\title{
Application of image recognition for plant virus detection
}

\author{
Min-Sheng Hung and Yi-Tzu Chiu
}

Department of Biomechatronic Engineering, National Chiayi University, Chiayi, Taiwan (Republic of China)

Agriculture contributes more than US\$1 trillion to the global economy every year. However, an estimated $20 \%-30 \%$ of agricultural products are lost annually due to plant disease. To overcome this problem, developing novel strategy for detecting plant pathogens is necessary. Pathogens of plant diseases include animals (e.g., birds and insects), plants (e.g., weeds), fungi, bacteria, and viruses. Diseases caused by fungi, bacteria, and viruses can inflict especially heavy losses on crops due to difficulties in their prevention. This study develops a magnetic-based microfluidic platform integrated with smartphone for detecting tomato yellow leaf curl virus (TYLCV). Conventionally, plant diseases are diagnosed either by conducting direct laboratory-based examination of pathogen nucleic acids and proteins, which involves nucleic acid testing and immunoassay [1], or by indirect methods such as monitoring the volatile organic compounds released in the metabolism of infected plants [2]. Although these methods can be used to accurately detect pathogens, plants in an early stage of infection (containing a scarce amount of virus) and small specimens (e.g., seeds) are challenging. Furthermore, PCR replication of pathogen DNA is ill-suited for onsite operations. Compared with conventional biochemical assays, a microfluidic system allows direct binding of the specimens' biochemical reactions on simplified microchips. This makes analysis and testing quicker and more efficient while consuming fewer specimens and reagents. In recent years, the pursuit of high sensitivity has led to the use of nanoparticle specificity to capture test targets. This approach has been successful in detecting small amounts of plant pathogens [3, 4]. One fluorescent labelling technique that can be applied is the use of quantum dots (QDs), which have narrow emission spectra and stable fluorescent luminance. Additionally, detecting QD fluorescent luminance with a fluorescence microscope is simple and highly sensitive $[5,6]$. To detect plant virus, we used magnetic beads to be evenly mixed and virus gene sequences captured in the microchannels. Under the influence of fluid dynamics in the microchannels and an external magnetic field, the magnetic beads that were bound with specimen gene sequences were directly condensed and aggregated in the microchannels. The experimental design is demonstrated in Figure 1. The specimen solution was guided into the reaction area in the microfluidic device. Because TYLVC is a begomovirus that contains circular single-stranded DNA molecules in its particle, the 5'-primer must be modified with biotin before replication to facilitate binding with the virus gene sequence. The product after replication (biotinylated primers) was bound with magnetic beads (probes) complementing target gene sequences to capture the biotin-carrying gene sequences. Subsequently, Sylgard 184 silicone elastomer base (Dow Corning) and Sylgard 184 silicone curing agent (Dow Corning) were used for the casting of a polydimethylsiloxane (PDMS) microfluidic device [5]. Figures 2 shows the fabricated microfluidics. In the upside of Fig. 2, the magnetic beads (probes) were injected into the microchannels and aggregated on the wall of microchannels under external magnet used. This result enabled the capture of target DNA in the microchannels. In addition, this study developed app detection software that uses OpenCV and image preprocessing to filter excess background noise to label fluorescence of plant virus gene. The techniques developed in this study can be applied to targeted gene detection for onsite operations. 
Biotinylated MPs with probe for products products capture

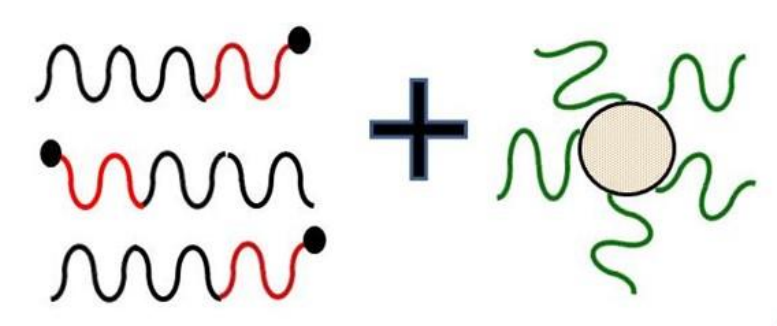

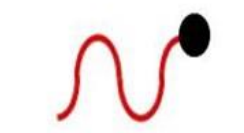

Primer with biotin labeled

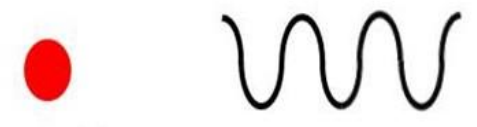

Replicatedvirus gene sequence

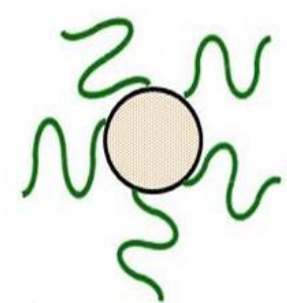

Magnetic particle with probe

Figure 1. Schematic diagram of magnetic beads for replication and capture viral gene sequences.

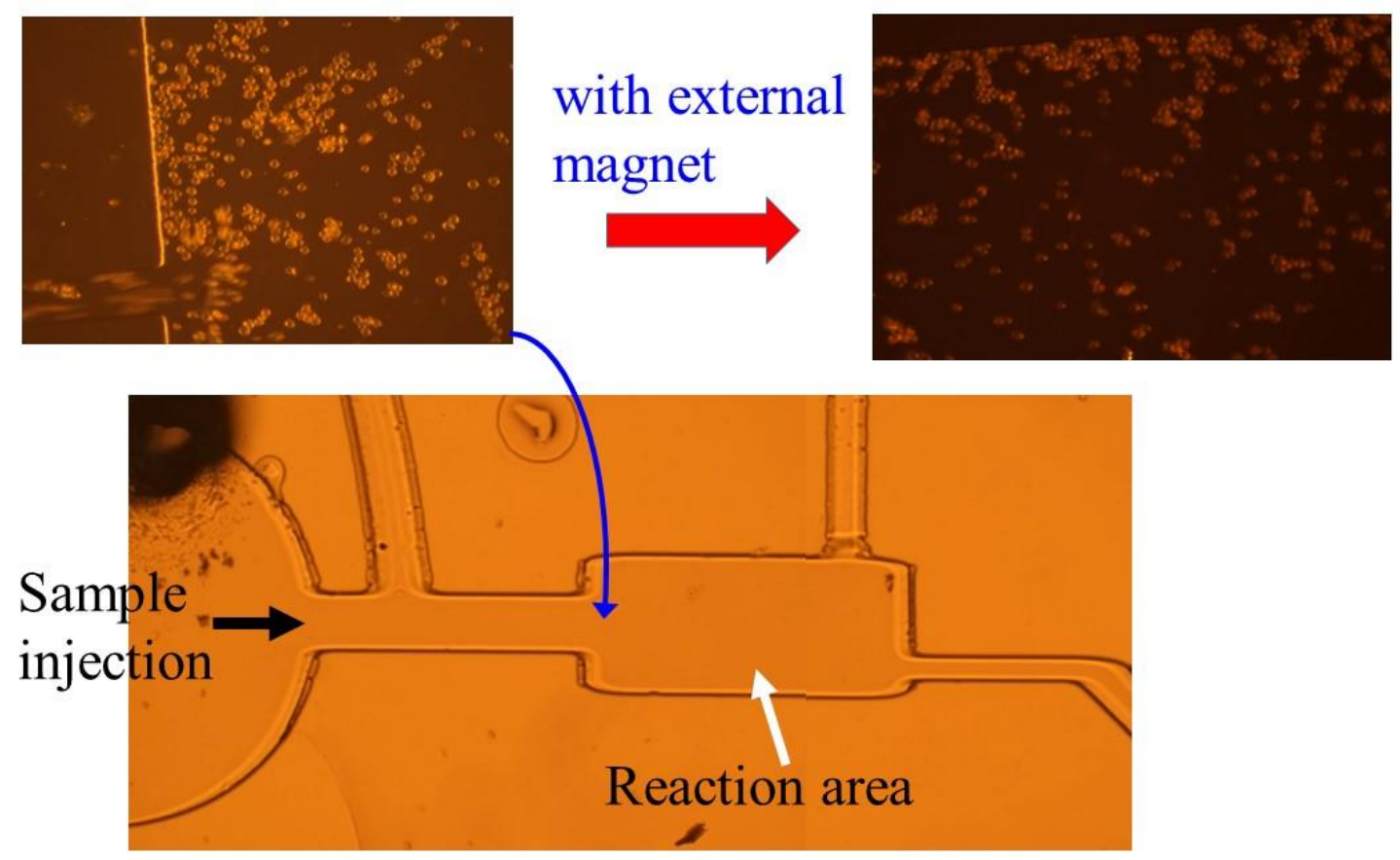

Figure 2. The images of fabricated microfluidics and magnetic beads. The diameter of magnetic bead is $10 \mu \mathrm{m}$. 


\section{References}

[1] López, M. M., Bertolini, E., Olmos, A., et al., Innovative tools for detection of plant pathogenic viruses and bacteria, Int. Microbiol. 6 (2003) 233-243.

[2] Scala, A., Allmann, S., Mirabella, R., et al., Green leaf volatiles: a plant's multifunctional weapon against herbivores and pathogens, Int. J. Mol. Sci. 14 (2013) 17781-17811.

[3] Kashyap, P. L., Kumar, S., Srivastava, A. K., Nanodiagnostics for plant pathogens, Environ. Chem. Lett. 15 (2017) 7-13.

[4] Wang, Y. and Duncan, T. V., Nanoscale sensors for assuring the safety of food products, Curr. Opin. Biotech. 44 (2017) 74-86.

[5] M.-S. Hung, C.-C. Ho, C.-P. Chen, Laser-induced heating integrated with a microfluidic platform for real-time DNA replication and detection, J. Biomed. Opt. 21 (2016) 087003.

[6] M.-S. Hung, C.-P. Chen, Laser-induced heating for in situ DNA replication and detection in microchannels, IET Nanobiotechnol. 12 (2018) 841-845. 